\title{
Aplicación del concepto de valor presente neto como técnica para evaluar el desempeño económico de los países*
}

\author{
José Gerardo de la Vega Meneses*
}

Recibido: 13 de mayo de 2012

Aprobado: 17 de junio de 2012

\begin{abstract}
Vega, J. de la (2012). Aplicación del concepto de valor presente neto como técnica para evaluar el desempeño económico de los países. Activos, 19, 41-66.
\end{abstract}

\section{JEL G00, E01}

\section{Resumen}

Esta investigación aplica la filosofía del valor presente neto para construir un indicador útil en materia de evaluación de desempeño económico de los países, agregando valor en cualquier toma de decisiones en materia de negocios internacionales. Este indicador se denomina valor presente neto per capita. Para este objetivo resulta fundamental el uso de variables económicas estratégicas implicadas en el cálculo del valor presente neto, como la consideración de la inversión inicial, flujo periódico, tasa de descuento, tasa de crecimiento y tasa de inflación, sustentando firmemente los orígenes de estas variables y sus fuentes de información, así como la conveniencia de su uso para ser aplicadas al modelo de medición en cuestión.

\footnotetext{
* Artículo de reflexión, derivado de proyecto de investigación "El concepto de valor presente neto y su aplicación en la medición del desempeño de los países", desarrollado como docente de la UPA del Estado de Puebla (tipo II).

${ }^{*}$ Profesor investigador de la Universidad Popular Autónoma del Estado de Puebla, México. Correo electrónico: josegerardo.delavega@upaep.mx
} 
Se realiza el cálculo del valor presente neto per capita en las principales economías del mundo y para ello se seleccionaron como muestra representativa los países de las siguientes zonas económicas y que conglomeran al motor económico mundial: la región de la Cooperación Económica de Asia Pacífico, la Unión Europea y, adicionalmente, las economías de Colombia, Brasil, India, Sudáfrica, Venezuela e Irán.

Finalmente, se determina el valor del valor presente neto per capita de la muestra representativa de países seleccionados y se establece un ranking que ilustra, acorde con el enfoque propuesto, una visión del desempeño económico de los países objeto de análisis, interpretando la tendencia de este indicador desde 2009 hasta 2011.

\title{
Palabras clave:
}

valor presente neto per capita, ranking, países.

\section{JEL Classification G00, E01}

Vega, J. de la (2012). Application of the net present value concept as a technique to evaluate the economic performance of the countries. Activos, 19, 41-66

\begin{abstract}
This research applies the net present value philosophy to build a useful indicator for the evaluation of a country's economic performance, adding value in any decision-making in international business matters. This indicator is called the net present value per capita. For this purpose it is essential to use strategic economic variables involved in the calculation of net present value, as consideration of the initial investment, periodic flow, discount rate, growth rate and inflation rate, supporting strongly the origins of these variables and their sources of information as well as the appropriateness of their use to be applied to the measurement model in question.
\end{abstract}


A calculation is made of the net present value per capita in the major economies of the world and for that a representative sample was selected of the following countries and economic zones which congregate the global economic engine: the region of Asia-Pacific Economic Cooperation, the European Union and, in addition, the economies of Colombia, Brazil, India, South Africa, Venezuela and Iran.

Finally, it is determined the value of the net present value per capita of the representative sample of selected countries and a ranking is established showing, according to the proposed approach, an overview of the economic performance of the countries under analysis, interpreting the trend of this indicator from 2009 to 2011.

\section{Keywords:}

net present value per capita, ranking, countries.

\section{Vega, J. de la (2012). Application du concept valeur actuelle nette pour évaluer la performance économique des pays. Activos, 19, 41-66}

\section{Résumé}

Cette recherche applique la philosophie de la valeur actuelle nette pour construire un indicateur utile en matière d'évaluation de la performance économique des pays, en ajoutant de la valeur à n'importe quelle prise de décision en matière d'affaires internationales. Cet indicateur est la valeur actuelle nette per capita. Pour cet objectif il est fondamental l'utilisation de variables économiques stratégiques intervenant dans le calcul de la valeur actuelle nette, comme la considération de l'investissement initial, le flux périodique, le taux dactualisation, le taux de croissance et le taux d'inflation, en soutenant fermement les origines de ces variables et des sources d'information ainsi que la pertinence de son utilisation pour être appliquées au modèle de mesure en question. 
Le calcul de la valeur actuelle nette per capita se fait dans les principales économies mondiales et pour cela les pays de zones économiques et qui conglomèrent le moteur économique mondial suivants ont été sélectionnées comme échantillon représentatif: la région de la Coopération Économique d'Asie Pacifique, l'Union Européenne et les économies de la Colombie, du Brésil, de l'Inde, de l'Afrique du Sud, du Venezuela et de l'Iran.

Finalement, la valeur de la valeur actuelle nette per capita est déterminée à partir de léchantillon représentatif des pays sélectionnés et un classement est établit ; celui-ci qui illustre, par rapport à l'approche proposée, une vision du rendement économique des pays objets d’analyse, en interprétant la tendance de cet indicateur de 2009 jusquen 2011.

\section{Mots-clés:}

valeur actuelle nette per capita, classement, pays.

\section{Introducción}

En el campo de la administración moderna, es constante el debate sobre la posibilidad de mejorar todo aquello que no es sujeto de medición. Chiang y Wainwright (2005) sostienen que en el estudio de las matemáticas es fundamental arribar a resultados relevantes que conlleven conclusiones y refuercen la toma de decisiones, siendo estratégica para la comprensión e interpretación de estos resultados la elaboración de gráficas, tablas o demostraciones.

En este sentido, Adam Smith (1979) sostenía en su conocida teoría laboral del valor o teoría del valor-trabajo que era este último la unidad exacta para cuantificar el valor de algo (Appleyard y Field, 2007). Además de la importancia de realizar mediciones que resulten en una propuesta de desarrollo de un indicador de desempeño económico, en el contexto internacional es relevante tener presente que los principales flujos de intercambio 
económico y financiero en las últimas décadas suceden especialmente en Europa, la región de Asia Pacífico y Norteamérica (Appleyard y Field, 2007).

Esto permite afirmar que la gran mayoría de las transacciones económico-financieras se efectúan en la región de la Cooperación Económica de Asia Pacífico mejor conocida como región APEC, en la Unión Europea $\mathrm{y}$, coincidentemente, las referidas regiones incluyen a los países que actualmente integran el G8+5, es decir, las ocho principales economías avanzadas más las cinco principales economías emergentes. Esta investigación propone un nuevo enfoque para el interesado en conocer la geografía económica mundial desde una nueva perspectiva consistente en la capacidad de generar valor.

\section{Marco teórico}

El concepto de valor presente neto (VPN) es definido por Ross, Westerfield y Jaffe (2011) como una técnica básica cuantitativa para toma de decisiones financieras, donde la fórmula para determinar el VPN para una inversión que genera flujos de efectivo en periodos futuros es

$$
V P N=-C_{0}+\frac{C}{(1+r)}+\frac{C}{(1+r)^{2}}+\cdots+\frac{C}{(1+r)^{N}}=-C_{0}+\sum_{t=1}^{N} \frac{C}{(1+r)^{t}}
$$

Donde:

VPN $=$ Valor presente neto o valor actual de los flujos de efectivo

$\mathrm{C}_{\mathrm{o}}=$ Inversión inicial

$\mathrm{C}=$ Flujo de efectivo por periodo

$r=$ Tasa de descuento de los flujos de efectivo

$\mathrm{t}=$ Número de periodos 
Por lo anterior, según Ross la fórmula del valor presente neto asume que el flujo de efectivo en el periodo cero es la inversión inicial (salida de dinero en caso de existir dicha inversión).

El cálculo del valor presente neto puede simplificarse aplicando las fórmulas de anualidades financieras, tanto constantes como crecientes, dependiendo del comportamiento de los flujos de efectivo futuros (Ross et al., 2011). Por esto, las fórmulas de anualidades son definidas por el referido autor de la siguiente forma:

$$
\begin{gathered}
V P N A c o=\frac{C}{r}\left[1-\frac{1}{(1+r)^{t}}\right]-C o \\
V P N A c o=\frac{C}{r}\left[1-\frac{1}{(1+r)^{t}}\right]-C o
\end{gathered}
$$

Donde:

VPNAco $=$ Valor presente neto de una anualidad constante

VPNAcr $=$ Valor presente neto de una anualidad creciente

Co = Inversión inicial

$\mathrm{C}=$ Flujo de efectivo por periodo

$\mathrm{r}=$ Tasa de descuento de los flujos de efectivo

$\mathrm{t}=$ Número de periodos

$\mathrm{g}=$ Tasa de crecimiento de los flujos de efectivo por periodo

Para perfeccionar el enfoque de valor presente neto es importante considerar el análisis de escenarios, examinando los resultados bajo distintas probabilidades de ocurrencia, donde la suma de todas ellas representa 
$100 \%$ (Ross et al., 2011). Dicho valor es considerado por Brealey, Myers y Marcus (2007) como la cantidad que se necesita invertir hoy para lograr obtener un pago futuro deseado, aspecto que representa la sumatoria de los flujos futuros esperados descontados apropiadamente menos el desembolso correspondiente a la inversión inicial.

En la determinación del valor presente neto es importante diferenciar entre flujos de efectivo reales y nominales, debido a que los flujos nominales deben convertirse en términos reales y eliminarse el efecto de la inflación. En este contexto, se recomienda expresar los flujos de efectivo futuros en términos reales o en paridad de poder adquisitivo y, de esta forma, eliminar el efecto de la inflación en los flujos futuros (Ross et al., 2011; Brealey et al., 2007).

Por otra parte, se requiere cuidado para distinguir la tasa de descuento nominal de la tasa de descuento real, donde la tasa real representa el valor sobre el cual la inversión crece, y se elimina el efecto inflacionario (Ross et al., 2011; Brealey et al., 2007), siendo imperativo descontar exclusivamente flujos nominales a tasas nominales y descontar solo flujos reales a tasas reales. Esta técnica de descuento de flujos de efectivo futuros es también considerada como la diferencia entre el valor presente de las entradas menos las salidas de dinero de un proyecto, en el cual este valor - siempre y cuando sea positivo - incrementará la riqueza de quien decida emprender el proyecto correspondiente (Higgings, 2007).

El valor presente neto es utilizado para determinar un momento de creación de valor. Nelson (2006) (citado en Kabay, 2006, p. 12) ha definido el término de valor presente neto de la Seguridad de la Información (VPNSeg) como:

la protección de valor y la creación de valor que es obtenida cuando las barreras a los e-business son eliminadas a través de mecanismos que aseguran la integridad del negocio, la disponibilidad en el servicio y, la privacidad y confidencialidad del cliente o consumidor. La creación de valor en este esquema incluye: nuevos canales de distribución, nuevos flujos de ingresos y efectivo $y$, nuevos modelos de negocios entre otros valores. 
Bacidore, Boquist, Milbourn y Thakor (1997) señalan que el VPN es un componente que, en adición al valor en libros de un activo, resulta en el valor de mercado de una empresa; por tanto, es sinónimo del valor de mercado de las oportunidades de inversiones, tanto actuales como futuras. Según Harris (1997), es también una técnica eficiente para medir el desempeño de una inversión, y afirma que esta valuación proporciona una visión referente a la capacidad de crear valor de la entidad a la cual se le ha aplicado este modelo.

Damodaran (2007) sostiene que cualquier técnica de valuación y medición que involucre indicadores financieros y económicos no puede omitir información referente al desempeño actual y esperado en el futuro del activo o ente valuado. Asimismo, afirma que en la tarea para perfeccionar cualquier técnica de valuación nada está escrito, pero para su desarrollo es deseable contar con una mezcla de habilidades interdisciplinarias que incluyan el conocimiento de técnicas contables, de estrategias y toma de decisiones destinadas a crear valor y el dominio de la estadística y de temas relacionados con las finanzas corporativas.

Al indagar más acerca de estudios relativos a modelos de valuación financiera y económica que se han elaborado por diversos investigadores y como información relevante del presente marco teórico, cabe destacar el análisis que Lambert y Burduroglu (2000) han establecido en relación con las principales ventajas y desventajas de diversos métodos o modelos existentes para medir o establecer el valor de algo en términos financieros. Así pues, se concluye en todos los casos analizados por los citados autores que el modelo de valuación más eficiente es aquel que reconoce el valor del dinero en el tiempo y que coincide con la metodología del Valor Presente Neto, lo cual subsana deficiencias que poseen diversas metodologías de valuación que se sustentan en variables cualitativas y enfocadas a la medición de la calidad.

No obstante, en métodos de valuación no se ha dicho la última palabra y es por esto que, en adición a lo anteriormente expuesto, se procederá 
a discutir respecto a tres posibles opciones reales como metodologías alternativas para el diseño del indicador VPN per capita propuesto.

\section{Primera opción alternativa: valor razonable}

Este término es definido por la Norma Internacional de Contabilidad Número 16 referente a Propiedades, Planta y Equipo (IASB, 2012) como "el importe por el cual podría ser intercambiado un activo, o cancelado un pasivo, entre partes interesadas y debidamente informadas, en una transacción realizada en condiciones de independencia mutua". Esta opción de valuación podría llegar a considerarse subjetiva, dado que depende en gran medida de la libertad que gozan las partes involucradas en la operación.

\section{Segunda opción alternativa: modelo binomial de valuación de opciones}

Este modelo, establecido para valuar opciones y desarrollado por Cox, Ross y Rubinstein (citados en Mascareñas, 2000), tiene la ventaja de ser muy intuitivo, y además utiliza una matemática muy simple. La limitante estriba en que es sencillo al usarse solamente para un periodo, pues es complicado extender su uso a varias etapas. Por tanto, realizar el diseño del indicador VPN per capita utilizando el modelo binomial de valuación de opciones resulta complicado, así como extenderlo a varios periodos.

\section{Tercera opción alternativa: modelo black-scholes de valuación de opciones}

Este modelo es utilizado para valuar opciones en términos financieros. Cabe destacar que necesita determinarse en su cálculo la probabilidad acumulada como función de la distribución normal estándar de algunos datos. Sin embargo, aunque es una alternativa de valuación vanguardista, debido 
a que en el estudio de las finanzas es ampliamente reconocido para valuar opciones, para efectos de lograr el objetivo de la presente investigación resultaría impráctico dada de la complejidad de las variables que utiliza, ya que se necesitarían datos longitudinales de varios años para determinar la desviación estándar del indicador económico que represente al flujo de efectivo periódico por considerar. De esta manera, dicha información resulta totalmente innecesaria desde la metodología del VPN.

En resumen, las tres metodologías alternativas de valuación citadas no proporcionan el mismo resultado y, sin lugar a dudas, la metodología del Valor Presente Neto representa la aproximación más real de creación de valor en un ente económico, debido a que muestra el valor presente de determinados flujos periódicos futuros representados en esta propuesta de medición, por el PIB per capita real de cada país objeto de estudio.

Por lo anterior, el modelo de valuación económica del Valor Presente Neto representa una técnica eficiente de medición; no obstante, su dificultad radica en el establecimiento de las variables que se utilizarían para estos fines (Lambert y Burduroglu, 2000).

\section{Modelo de estudio}

Para calcular el valor presente neto se utilizan los métodos del valor presente de las anualidades con base en dos escenarios: anualidades constantes y anualidades crecientes, considerándose las mismas posibilidades de ocurrencia. A su vez, se destaca que el referido modelo cuenta con la característica de reconocer el valor del dinero en el tiempo, los riesgos asociados y, en general, poseer la ventaja de eliminar ineficiencias de medición que otros modelos de valuación financiera tienen (Lambert y Burduroglu, 2000).

El razonamiento del modelo por utilizar (tabla 1) es el siguiente: el VPN per capita es equivalente al valor presente neto de una anualidad contingente (considerando probabilidades de ocurrencia), cuyo flujo 
financiero periódico está representado por el PIB per capita real anual, y tomando como espacio temporal el índice de esperanza de vida. La tasa de descuento real está representada por la tasa libre de riesgo de los bancos centrales descontándose el efecto de la inflación, y la tasa de crecimiento requerida para el cálculo está representada por la tasa de crecimiento en PIB per capita. Por su parte, la inversión inicial para el desarrollo de este indicador está representada por la deuda externa per capita.

Tabla 1: Modelo de estudio: Diseño del indicador de desempeño económico VPN per capita o VPN per capita

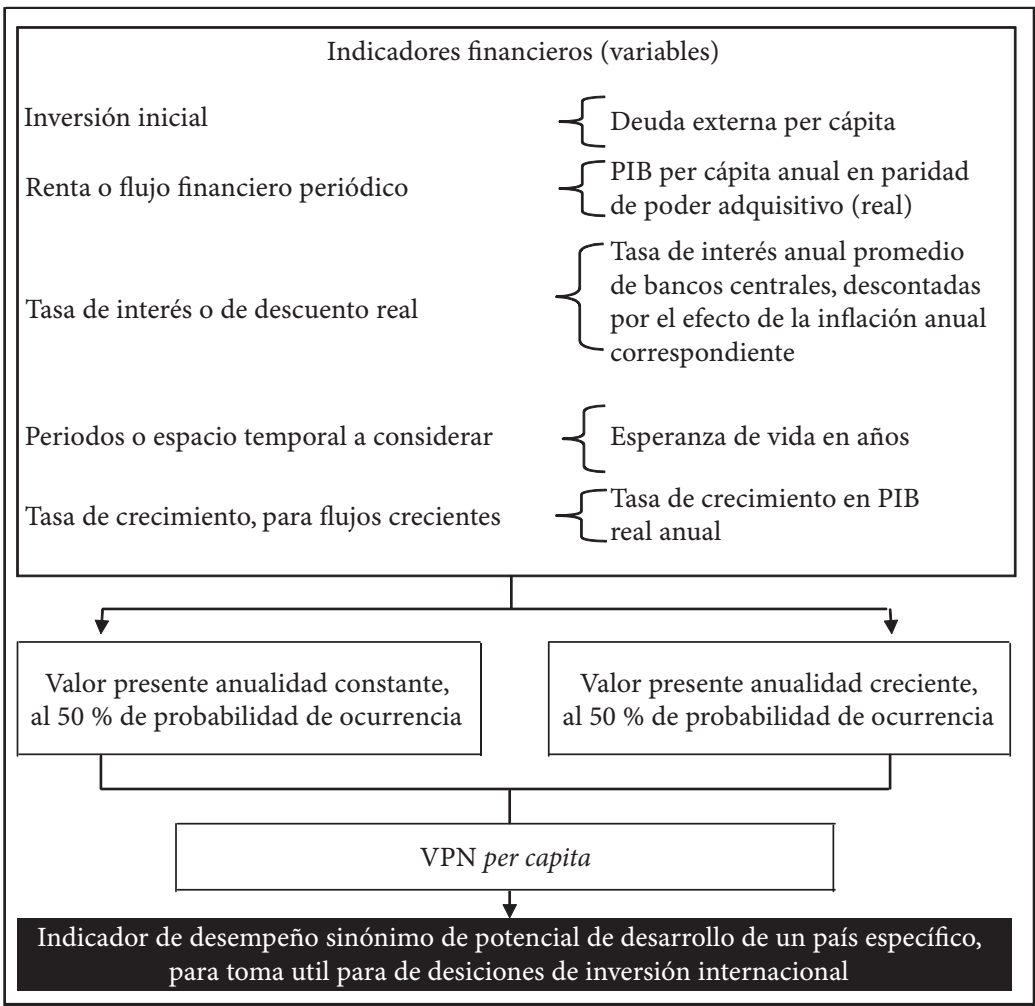

La valoración que se presenta a continuación considerará a las principales economías del mundo, las cuales abarcan los países pertenecientes a la Cooperación Económica de Asia Pacífico, región que representa la mitad 
del comercio mundial, $41 \%$ de la población mundial y casi $57 \%$ del PIB mundial (Heseltine, 2007); los países de la Unión Europea y, adicionalmente por ser economías emergentes de gran protagonismo internacional, los países de Colombia, Brasil, India, Sudáfrica, Venezuela e Irán.

\section{Metodología}

Para el presente análisis se diseñó una aplicación con las siguientes características.

\section{Variables utilizadas}

Se realizó una investigación no experimental en la cual se obtuvieron las variables después de ocurridos los hechos y en la que se utilizaron datos históricos de los años 2009 a 2011.

\section{Fuentes de información}

Se realizó una investigación documental utilizando fuentes de información de bases de datos de alto reconocimiento internacional y $100 \%$ verificables.

\section{Extensión del estudio}

Se realizó una investigación longitudinal utilizando datos de los años más recientes disponibles, con objeto de obtener resultados a corto plazo y que deberán ir cambiando año por año según la variación en los indicadores financieros establecidos en el modelo. En esta medición se recolectaron datos en tres periodos, con el propósito de describir variables y analizar su incidencia e interpretación en tres momentos determinados. 


\section{Nivel de medición y análisis de la información}

Se realizó una investigación descriptiva calculando el VPN per capita en las principales economías para determinar a través de este indicador el potencial desarrollo económico en los países; se obtuvieron conclusiones al respecto.

En el desarrollo del indicador VPN per capita se requirió, según lo propuesto en el modelo de estudio, contar con los siguientes indicadores financieros:

1. Inversión inicial. La fuente secundaria de investigación de este indicador fue la Agencia Central de Inteligencia Norteamericana (CIA, 2012). Se consiguió información referente a la deuda externa de cada uno de los países muestra y se dividió el valor entre el número de habitantes respectivo, para obtener el dato a nivel per capita.

2. Renta o flujo financiero periódico. La fuente secundaria de investigación de este indicador fue la Agencia Central de Inteligencia Norteamericana (CIA, 2012). Se obtuvieron los datos de cada uno de los países seleccionados referentes al PIB per capita anual en dólares en paridad de poder adquisitivo (indicador en términos reales).

Tasa de interés o de descuento real. Para la obtención de este indicador, acorde con la teoría Fisher, las tasas reales se logran descontado a las tasas de interés base o nominales la inflación, siempre y cuando los bancos centrales de los países modifiquen su tasa de interés en función de la inflación (Tymoigne, 2006). Por tanto, una parte la fuente secundaria de investigación correspondiente a la tasa de inflación en este indicador fue la Agencia Central de Inteligencia Norteamericana (CIA, 2012), de la cual se alcanzaron los referidos datos correspondientes a 2009, 2010 y 2011 de los países seleccionados en el presente análisis. Por otra parte, las tasas de interés nominales bases se obtuvieron para cada país de la siguiente forma: 
1. Estados Unidos. Se obtuvo la tasa libre de riesgo de la Reserva Federal Norteamericana a diciembre de los referidos años (Bloomberg, 2012).

2. Países no asiáticos. Se adquirieron las tasas de interés promedio libre de riesgo anual publicadas como parte de los indicadores económicos claves de las economías pertenecientes a la Cooperación Económica de Asia Pacífico (APEC, 2012).

3. Países asiáticos. Se consiguieron las tasas de interés promedio libre de riesgo anual publicadas en la base de datos del Banco Asiático de Desarrollo (ADB, 2012).

4. Países de la Unión Europea. Se obtuvieron las tasas de interés promedio libre de riesgo anual publicadas en la base de datos del Banco Central Europeo (BCE, 2012).

5. Colombia, Brasil, Venezuela, Sudáfrica e Irán. Se lograron las tasas anuales objetivo de política monetaria establecidas a diciembre de 2009 a 2011 por sus respectivos bancos centrales (Banco de la República, 2012; Bloomberg, 2012b; BCV, 2012; SARB, 2012; CBIRI, 2012).

\section{Periodos o espacio temporal por considerar}

La fuente secundaria de investigación de este indicador fue la Agencia Central de Inteligencia Norteamericana (CIA, 2012). Se obtuvieron los datos de los países seleccionados en referencia a la esperanza de vida en años de sus habitantes.

\section{Tasas de crecimiento para flujos crecientes}

La fuente secundaria de investigación de este indicador fue la Agencia Central de Inteligencia Norteamericana (CIA, 2012). Se consiguieron los 
datos de los países seleccionados referentes a las tasas de crecimiento anual reales en PIB.

El valor presente neto per capita se obtuvo sustituyendo las variables identificadas a través de las fuentes secundarias de investigación en la ecuación a la que hace referencia el modelo, que en esencia corresponde a la sumatoria del valor presente de una anualidad constante (es decir, crecimiento nulo) y una anualidad creciente con el mismo porcentaje de probabilidad de ocurrencia. De esta manera, se alcanzó el valor presente de todos los flujos futuros por persona que representan los PIB per capita en cada uno de los países seleccionados de este análisis.

\section{Resultados obtenidos}

Una vez sustituidos los indicadores financieros en el modelo de estudio desarrollado en este análisis y sustentado con el marco teórico expuesto con anterioridad, se presenta el VPN per capita correspondiente a 2011 (tabla 2). Allí se destacan, entre otros, los siguientes resultados acordes con los indicadores financieros sustituidos en el referido modelo.

Los actualmente considerados países ricos o industrializados reflejan un VPN per capita superior al millón de dólares, y se encuentra la única excepción en el caso de Grecia, país que no logró superar la barrera referida. En este contexto, cabe resaltar que Portugal y Nueva Zelanda solo superaron ligeramente este monto del millón de dólares en VPN per capita.

El único país que obtuvo un VPN per capita superior a los diez millones de dólares fue China, como resultado del registro de tasas de crecimiento económico anual en términos de PIB superiores a $7 \%$, una satisfactoria expectativa de vida entre sus habitantes y una variación modesta entre la tasa de interés de su banco central y su tasa de inflación (es decir, contó con tasa de descuento real muy baja). No obstante, China no es un país de alta renta per capita actualmente. 
Los países que no lograron en 2011 superar - o superaron por escaso margen - el límite de un millón de dólares en VPN per capita acorde con el modelo propuesto fueron aquellos con desempeño económico no satisfactorio: Hungría, Grecia, Malta, Rumania y Sudáfrica. En este sentido, cabe destacar que los países que registraron las menores mediciones de VPN cápita acorde con el modelo propuesto manifestaron los siguientes factores comunes: el PIB per capita a niveles muy por debajo de los países desarrollados y las tasas de crecimiento anual en PIB muy deficientes; incluso, representaron en algunos casos un acentuado decrecimiento económico o tasas de crecimiento negativas, como sucedió en los casos de Grecia y Hungría. En cuanto a Sudáfrica, este indicador se ve castigado notablemente por la deficiente esperanza de vida de su población, que no supera los 50 años. El resumen de resultados se encuentra en la tabla 2.

Tabla 2. VPN per capita (2011)

\begin{tabular}{|c|c|c|c|c|c|}
\hline $\begin{array}{c}\text { País/ } \\
\text { economía }\end{array}$ & $\begin{array}{c}\text { Valor } \\
\text { presente } \\
\text { anualidad } \\
\text { constante } \\
(\$)\end{array}$ & $\begin{array}{c}\text { Ponderación } \\
\text { (probabilidad) } \\
(\%)\end{array}$ & $\begin{array}{c}\text { Valor } \\
\text { presente } \\
\text { anualidad } \\
\text { creciente } \\
(\$)\end{array}$ & $\begin{array}{c}\text { Ponderación } \\
\text { (probabilidad) } \\
(\%)\end{array}$ & $\begin{array}{c}\text { VPN per } \\
\text { capita } \\
(\$)\end{array}$ \\
\hline \multicolumn{6}{|l|}{ Zona APEC } \\
\hline Australia & 2170550 & 50 & 8976005 & 50 & 5573278 \\
\hline Brunei & 3851661 & 50 & 12404129 & 50 & 8127895 \\
\hline Canadá & 3341973 & 50 & 7885180 & 50 & 5613577 \\
\hline Chile & 1430466 & 50 & 16240133 & 50 & 8835299 \\
\hline China & 641530 & 50 & 28600475 & 50 & 14621002 \\
\hline Hong Kong & 4033530 & 50 & 9236394 & 50 & 6634962 \\
\hline Indonesia & 193269 & 50 & 1918868 & 50 & 1056069 \\
\hline Japón & 2772341 & 50 & 7671284 & 50 & 5221813 \\
\hline Corea del & 2559299 & 50 & 8711074 & 50 & 5635186 \\
\hline \multicolumn{6}{|l|}{ Sur } \\
\hline Malasia & 911983 & 50 & 5583222 & 50 & 3247603 \\
\hline México & 1011684 & 50 & 5362377 & 50 & 3187030 \\
\hline
\end{tabular}




\begin{tabular}{|c|c|c|c|c|c|}
\hline $\begin{array}{c}\text { País/ } \\
\text { economía }\end{array}$ & $\begin{array}{c}\text { Valor } \\
\text { presente } \\
\text { anualidad } \\
\text { constante } \\
(\$)\end{array}$ & $\begin{array}{c}\text { Ponderación } \\
\text { (probabilidad) } \\
(\%)\end{array}$ & $\begin{array}{c}\text { Valor } \\
\text { presente } \\
\text { anualidad } \\
\text { creciente } \\
(\$)\end{array}$ & $\begin{array}{c}\text { Ponderación } \\
\text { (probabilidad) } \\
(\%)\end{array}$ & $\begin{array}{l}\text { VPN per } \\
\text { capita } \\
(\$)\end{array}$ \\
\hline Nueva & 723299 & 50 & 1325377 & 50 & 1024338 \\
\hline \multicolumn{6}{|l|}{ Zelanda } \\
\hline \multicolumn{6}{|l|}{ Papúa } \\
\hline Nueva & 144045 & 50 & 3376022 & 50 & 1760033 \\
\hline \multicolumn{6}{|l|}{ Guinea } \\
\hline Perú & 723803 & 50 & 10887766 & 50 & 5805784 \\
\hline Filipinas & 298579 & 50 & 2401976 & 50 & 1350277 \\
\hline Rusia & 560531 & 50 & 1638332 & 50 & 1099431 \\
\hline Singapur & 5108864 & 50 & 13705308 & 50 & 9407086 \\
\hline Tailandia & 737997 & 50 & 9881991 & 50 & 5309994 \\
\hline Estados & 3885780 & 50 & 10314952 & 50 & 7100366 \\
\hline \multicolumn{6}{|l|}{ Unidos } \\
\hline Vietnam & 254948 & 50 & 2520690 & 50 & 1387819 \\
\hline \multicolumn{6}{|l|}{ No Zona } \\
\hline \multicolumn{6}{|l|}{ APEC } \\
\hline India & 260963 & 50 & 2375346 & 50 & 1318154 \\
\hline Colombia & 658457 & 50 & 9655318 & 50 & 5156887 \\
\hline Brasil & 583150 & 50 & 1553597 & 50 & 1068374 \\
\hline Sudáfrica & 176865 & 50 & 264862 & 50 & 220864 \\
\hline Irán & 921791 & 50 & 1984558 & 50 & 1453175 \\
\hline Venezuela & 455802 & 50 & 3829404 & 50 & 2142603 \\
\hline \multicolumn{6}{|l|}{ Euro Zona } \\
\hline Bélgica & 2882552 & 50 & 3017072 & 50 & 2949812 \\
\hline Alemania & 3057558 & 50 & 4415649 & 50 & 3736603 \\
\hline Irlanda & 2302020 & 50 & 3609381 & 50 & 2955700 \\
\hline Grecia & 1511469 & 50 & -124183 & 50 & 693643 \\
\hline España & 2415822 & 50 & 1378552 & 50 & 1897187 \\
\hline \multirow[t]{2}{*}{ Francia } & 2802246 & 50 & 2953065 & 50 & 2877656 \\
\hline & & & & & Continu: \\
\hline
\end{tabular}




\begin{tabular}{|c|c|c|c|c|c|}
\hline $\begin{array}{c}\text { País/ } \\
\text { economía }\end{array}$ & $\begin{array}{c}\text { Valor } \\
\text { presente } \\
\text { anualidad } \\
\text { constante } \\
(\$)\end{array}$ & $\begin{array}{c}\text { Ponderación } \\
\text { (probabilidad) } \\
(\%)\end{array}$ & $\begin{array}{c}\text { Valor } \\
\text { presente } \\
\text { anualidad } \\
\text { creciente } \\
(\$)\end{array}$ & $\begin{array}{c}\text { Ponderación } \\
\text { (probabilidad) } \\
(\%)\end{array}$ & $\begin{array}{l}\text { VPN per } \\
\text { capita } \\
(\$)\end{array}$ \\
\hline Italia & 2417957 & 50 & 1026573 & 50 & 1722265 \\
\hline Chipre & 2000553 & 50 & 938634 & 50 & 1469593 \\
\hline Luxemburgo & 2245543 & 50 & 6893874 & 50 & 4569708 \\
\hline Malta & 1968155 & 50 & -736394 & 50 & 615880 \\
\hline Países Bajos & 3265952 & 50 & 4090465 & 50 & 3678208 \\
\hline Austria & 3291011 & 50 & 4186611 & 50 & 3738811 \\
\hline Portugal & 1765030 & 50 & 344306 & 50 & 1054668 \\
\hline Eslovenia & 2182157 & 50 & 1016087 & 50 & 1599122 \\
\hline Finlandia & 2788067 & 50 & 3234899 & 50 & 3011483 \\
\hline Eslovaquia & 1833726 & 50 & 5630823 & 50 & 3732275 \\
\hline \multicolumn{6}{|l|}{ No Euro } \\
\hline \multicolumn{6}{|l|}{ Zona } \\
\hline Bulgaria & 1044229 & 50 & 1544660 & 50 & 1294445 \\
\hline República & 2095614 & 50 & 1463829 & 50 & 1779721 \\
\hline \multicolumn{6}{|l|}{ Checa } \\
\hline Dinamarca & 2863127 & 50 & 2548157 & 50 & 2705642 \\
\hline Letonia & 925917 & 50 & 5657094 & 50 & 3291506 \\
\hline Lituania & 1495956 & 50 & 4713081 & 50 & 3104519 \\
\hline Hungria & 920311 & 50 & 693179 & 50 & 806745 \\
\hline Polonia & 1587308 & 50 & 4412272 & 50 & 2999790 \\
\hline Rumania & 405421 & 50 & 515409 & 50 & 460415 \\
\hline Suecia & 3273000 & 50 & 5663970 & 50 & 4468485 \\
\hline Reino Unido & 2786018 & 50 & 2716982 & 50 & 2751500 \\
\hline Estonia & 1548273 & 50 & 4067350 & 50 & 2807812 \\
\hline
\end{tabular}

Basados en los resultados obtenidos, es importante resaltar las diez economías o países mejor posicionados en cuanto al modelo de valuación 
propuesto, y mostrar adicionalmente sus tasas de crecimiento anual en PIB para corroborar que son economías que aún siguen creciendo, aunque modestamente. Por esta razón, se afirma que el desarrollo económico reciente de estos países es de los más satisfactorios, a pesar de la actual situación de crisis mundial (tabla 3 ).

Tabla 3. Países identificados con el desarrollo económico actual más satisfactorio acorde al indicador VPN per capita (2011)

\begin{tabular}{|c|c|c|c|c|c|}
\hline $\begin{array}{c}\text { Ranking } \\
2009\end{array}$ & $\begin{array}{c}\text { Ranking } \\
2010\end{array}$ & $\begin{array}{c}\text { Ranking } \\
2011\end{array}$ & $\begin{array}{c}\text { País / } \\
\text { Economía }\end{array}$ & $\begin{array}{c}\text { Valor presente } \\
\text { neto per capita } \\
\text { en dólares (2011) }\end{array}$ & $\begin{array}{l}\text { Tasa real de creci- } \\
\text { miento anual en PIB } \\
\text { (2011) }\end{array}$ \\
\hline 1 & 1 & 1 & China & $\$ 14621002$ & $7,800 \%$ \\
\hline 4 & 3 & 2 & Singapur & 9407086 & $2,100 \%$ \\
\hline 12 & 8 & 3 & Chile & 8835299 & $5,000 \%$ \\
\hline 14 & 10 & 4 & Brunéi & 8127895 & $2,700 \%$ \\
\hline 20 & 13 & 5 & $\begin{array}{l}\text { Estados } \\
\text { Unidos }\end{array}$ & 7100366 & $2,200 \%$ \\
\hline 7 & 7 & 6 & Hong Kong & 6634962 & $1,800 \%$ \\
\hline 5 & 9 & 7 & Perú & 5805784 & $6,000 \%$ \\
\hline 13 & 11 & 8 & $\begin{array}{l}\text { Corea del } \\
\text { Sur }\end{array}$ & 5635186 & $2,700 \%$ \\
\hline 16 & 12 & 9 & Canadá & 5613577 & $1,900 \%$ \\
\hline 15 & 14 & 10 & Australia & 5573278 & $3,300 \%$ \\
\hline
\end{tabular}




\section{Resultados del ejercicio: economías resistentes, economías en dificultades y los casos de los países latinoamericanos}

El VPN per capita más satisfactorio durante 2011 lo manifestaron China, Singapur, Chile, Brunei y Estados Unidos. Estos representan a los países con desempeño económico favorable en el referido año a pesar de la persistencia de la crisis económica mundial; no obstante, algunos de estos países no registraron una elevada tasa de crecimiento, siendo los crecimientos de China en PIB los más notables en este caso (7,8 \%) y los Chile (5\%).

China logró el VPN per capita más alto como consecuencia de es un país con una deuda externa per capita muy baja, y registró una destacada tasa de crecimiento económico (7,8 \%), a pesar de ser un país cuyo PIB per capita no supera los $\$ 10000$ dólares. A la luz del VPN per capita, China resultó la economía con mayor potencial para generar valor económico por habitante. Por otra parte, el indicador registrado por Singapur está asociado principalmente al hecho de poseer un alto PIB per capita con valor ligeramente superior a los $\$ 60000$ dólares, aunado a que dicho país creció económicamente en 2011 un moderado 2,1 \%, crecimiento favorable considerando la manifestación de dificultades económicas que enfrenta la economía global.

En el caso de Chile, este registra un satisfactorio VPN per capita durante 2011, principalmente por registrar una tasa de crecimiento económico de alrededor de $5 \%$ que en el actual periodo de crisis, hecho que resulta más que satisfactorio. En adición, el PIB per capita de Chile es aceptable y está en camino a la senda de alcanzar niveles de países desarrollados, situándose en los $\$ 18400$ dólares. Con respecto a Brunei, este país alcanzó un satisfactorio VPN per capita durante 2011 como resultado de tener un alto PIB per capita que se ubica en un nivel superior a $\$ 50000$ dólares y poseer un moderado endeudamiento externo; no obstante, su tasa de crecimiento económico fue moderada al ubicarse este crecimiento en un 2,7 \% aproximadamente. Finalmente, Estados Unidos registró un satisfactorio VPN per 
capita en 2011, a pesar de que, al igual que en los casos de Singapur y Brunei, su tasa de crecimiento económico ha sido moderada en los últimos años al no superar $3 \%$ y en adición posee un endeudamiento externo elevado. Sin embargo, en el caso norteamericano se cuenta con un elevado nivel de PIB per capita, de $\$ 49800$ dólares, una expectativa de vida elevada y muy cercana a los 80 años, (superior a las de China, Chile y Brunei) y cuenta con bajas tasas de interés que son objetivo de su banco central y las cuales han contribuido a controlar la inflación.

Así pues, un VPN per capita satisfactorio tiene su explicación en los siguientes elementos: una economía sin problemas graves de deuda externa, con crecimiento económico satisfactorio o al menos en la senda de crecimiento sostenido, con tasas de interés de sus bancos centrales no muy distantes de las tasas de inflación y esperanzas de vida satisfactorias en sus habitantes. Esto resulta sin ser indispensable registrar altos niveles de PIB per capita, como sucedió en el caso de China.

Ahora bien, es interesante discutir los casos de Rumanía, Eslovenia, Bulgaria, Grecia e Irán, dado que representaron a los países cuyo indicador VPN per capita disminuyó drásticamente en comparación con los 53 países seleccionados durante en 2011 y en referencia a los dos años anteriores (2009 y 2010).

Rumanía, después de haber clasificado su VPN per capita en 2009 y 2010 en las primeras 20 posiciones, en 2011 su VPN per capita se posicionó en el número 52, como consecuencia de que su PIB per capita se ubica aún en niveles de economía en desarrollo al no superar los $\$ 13000$ dólares y al registrar tasas de interés y de inflación muy superiores a las promedio de los países de la Unión Europea; además, su tasa de crecimiento económico ha disminuido considerablemente al registrar apenas un 0,9\% en 2011. En el caso de Eslovenia, después de haber clasificado su VPN per capita en 2009 y 2010 en las primeras 15 posiciones, en 2011 su VPN per capita se posicionó en el número treinta y siete, como resultado de que, a pesar de que su PIB per capita se ha mantenido en niveles de países desarrollados 
al ubicarse en alrededor de $\$ 29000$ dólares, en 2011 registró una notable contracción económica de -2,2\%.

Con respecto a Bulgaria, después de haber clasificado su VPN per capita en 2009 y 2010 en las primeras 15 posiciones, en 2011 su VPN per capita se posicionó a la baja drásticamente hasta situarse en el número 43 , como consecuencia de que su PIB per capita aún se mantiene en niveles de economía en desarrollo al no superar los $\$ 15000$ dólares, su esperanza de vida por habitante es de las más bajas de la Unión Europea y su crecimiento económico fue muy moderado en 2011 al alcanzar solo $1 \%$. Con respecto a Grecia, después de haber clasificado su VPN per capita en 2009 dentro de las primeras 20 posiciones, en 2010 y 2011 su VPN per capita se posicionó a la baja drásticamente hasta situarse en la posición 50 , como consecuencia de que, no obstante su PIB per capita se ha mantenido sin amplias variaciones y es cercano a los \$25000 dólares en promedio y la esperanza de vida es de las más altas de la Unión Europea, su tasa de crecimiento económico ha disminuido considerablemente al registrar contracciones económicas de $-3 \%$ y $-6 \%$ durante 2010 y 2011. Lo anterior ha sido resultado de la delicada situación financiera del país, que ha requerido repetidas asistencias del Banco Central Europeo y el Fondo Monetario Internacional. Por último, en el caso de Irán, después de haber clasificado su VPN per capita en 2009 y 2010 incluso en la primeras diez posiciones, en 2011 su VPN per capita se posicionó a la baja drásticamente hasta situarse en el número 39, puesto que, no obstante su PIB per capita se ha mantenido sin amplias variaciones y es de alrededor de $\$ 13000$ dólares, es un país con elevada tasa de inflación al ser superior a $20 \%$, la expectativa de vida de sus habitantes supera apenas los 70 años y su crecimiento en PIB ha disminuido considerablemente en 2011 al registrar solo $2 \%$, mientras que en años anteriores esta había superado al menos $5 \%$.

Finalmente, es de resaltar que en el presente estudio se consideraron seis países latinoamericanos: Brasil, Chile, Colombia, México, Perú y Venezuela. De estos seis países, Chile, Perú y Colombia registraron los mayores niveles de VPN per capita al ser economías que han manifestado crecimientos en PIB satisfactorios al superar al menos $5 \%$ o ubicarse en una tasa similar, 
la expectativa de vida de sus habitantes es igualmente satisfactoria entre 73 y 78 años de vida, mantienen moderada la tasa de inflación oficial en un $3 \%$ o $4 \%$ aproximadamente y un moderado endeudamiento con el exterior. Por su parte, México, Venezuela y Brasil no superaron los niveles de VPN per capita de Chile, Perú y Colombia, principalmente como consecuencia de que el cálculo de este indicador, en el caso de Brasil, se vio disminuido por una inflación superior a $5 \%$ y una tasa de crecimiento en PIB moderada que no superó $3 \%$; en el caso de México por haber registrado una inflación superior a $4 \%$ y una tasa de crecimiento en PIB que no superó tampoco $4 \%$; en el caso de Venezuela, a pesar de que su economía ha crecido en niveles superiores a $5 \%$ en los últimos años, su elevada tasa de inflación se ubica en niveles superiores a $20 \%$, lo que influyó notablemente en el descuento de flujos futuros acorde con modelo de estudio aquí presentado.

Es por lo anterior que resultará interesante conocer la variación que se manifieste en 2012 y 2013 en el desempeño económico de los países a la luz del VPN per capita propuesto en la presente investigación, indicador que sin lugar a dudas reflejará las consecuencias surgidas como resultado del agravamiento de la crisis económica mundial en curso, siendo muy probable que China aún se mantenga en las primeras posiciones; aspecto que representa una muestra de su fortaleza económica del presente.

\section{Conclusiones}

Es viable el diseño del indicador VPN per capita como una medida de desempeño económico de los países, utilizando como indicadores financieros para su desarrollo los siguientes: la inversión inicial estará representada por la deuda externa a nivel per capita, el flujo monetario deberá expresarse en términos de PIB per capita real, la tasa de descuento de los referidos flujos (entendida como el riesgo) debe considerar el efecto de la inflación y los periodos por considerar tienen como su principal fundamento la esperanza de vida en años según la nacionalidad del país en cuestión. 
China representa a la gran potencia económica con mayor capacidad de crear valor por ciudadano, es decir, con el VPN per capita más elevado.

El VPN per capita es sinónimo del desempeño actual de la economía de un país. Su interpretación no debe ser muy distante de la realidad reflejada a través de la tasa de crecimiento anual en el PIB.

Un país cuyo VPN per capita es considerado bajo, es sinónimo de un país que posee desempeño económico deficiente, siendo las principales causas su deficiente tasa de crecimiento económico, poseer bajos niveles de PIB per capita, así como manifestar una brecha importante entre la tasa de interés de su banco central y la tasa de inflación (tasa real de descuento aplicada en el modelo).

El VPN per capita está sujeto a severa variación (riesgo) debido a ser fuertemente influenciado en su determinación por las tasas de crecimiento en PIB que anualmente manifiesten los países, tasas cuyo comportamiento es muy incierto en el corto plazo. Esta aseveración se dejó ver muy en claro en los casos de Grecia y Hungría, que han manifestado síntomas inequívocos de recesión económica desde 2010 y que han ocasionado caídas estrepitosas en este indicador objeto de estudio en comparación con los años 2009 y anteriores.

Finalmente, este modelo de valuación de desempeño económico es ampliamente dependiente de los indicadores financieros que deben aplicarse al sustituirse en la ecuación matemática objeto de estudio, basada en un cálculo de anualidades financieras. Por tanto, la idoneidad y confiabilidad de las fuentes de información de las cuales se extraigan los indicadores financieros del modelo son un elemento clave para la determinación de esta valuación, sinónimo de desempeño financiero y económico actual de un país. 


\section{Referencias}

Asian Development Bank (2012). Key Indicators for Asia and the Pacific 2007. Recuperado de http://www.adb.org/Documents/Books/Key_ Indicators/2007/default.asp

Asia Pacific Economic Cooperation (2012). Key Economic Indicators. Recuperado de http://aimp2.apec.org/sites/PDB/default.aspx

Appleyard, D. y Field, A. (2007). International economics. The world of international economics. Estados Unidos: McGraw-Hill.

Bacidore, J. M., Boquist, J. A., Milbourn T. T. y Thakor, A. V. (1997, mayo-junio). The search for the best financial performance measure. Financial Analysts Journal, 53, 11-20.

Banco Central Europeo (2012). Long term statistics. Recuperado de http://www. ecb.int/stats/money/long/html/index.en.html

Banco Central de Venezuela (2012). Calendario anticipado de divulgación. Estadísticas de tasas de interés anuales. Recuperado de http://www.bcv.org. ve/calendario/calanticipado.htm

Banco de la República, Colombia (2012). Indicadores. Tasa de intervención del Banco de la República. Recuperado de http://www.banrep.gov.co/es/seriesestadisticas/see_tas_intervencion.htm

Bloomberg (2012). Rates \& bonds market data USA. Recuperado de http://www. bloomberg.com/markets/rates/index.html

Bloomberg (2012b). Rates \& bonds market data Brazil. Recuperado de http:// www.bloomberg.com/markets/rates/brazil.html

Brealey, R. A., Myers, S. C. y Marcus, A. J. (2007). Fundamentals of Corporate Finance (5. ${ }^{\text {a }}$ ed.). Estados Unidos: McGraw-Hill.

CBIRI Central Bank of the Islamic Republic of Iran (2012). Private banking profits rates. Recuperado de http://www.cbi.ir/simplelist/1493.aspx

Chiang, A. y Wainwright, K. (2005). Fundamental methods of mathematical economics. Estados Unidos: McGraw-Hill.

Central Intelligence Agency (2012). The world factbook 2011. Recuperado de https://www.cia.gov/library/publications/the-world-factbook/index.html

Damodaran, A. (2007). Valuation approaches and metrics: A survey of the theory. Estados Unidos: Now Publishers. 
Harris, R. (1997). Value creation, net present value and economic profit; papel de trabajo identifcación UVA-F-1164. Virginia: Darden School of Business

Heseltine, C. (2007, 17 de diciembre). Discurso inaugural de la Conferencia "Global maritime and intermodal logistics 2007 conference". Singapur. Recuperado de http://www.apec.org/apec/news media/speeches/171207_sg_globalmartconfcsh.html

Higgins, R. C. (2007). Analysis for financial management (8. ${ }^{\mathrm{a}}$ ed.). Estados Unidos: McGraw-Hill.

International Accounting Standards Board (2012). Norma Internacional de Contabilidad Número 16: Propiedades, planta y equipo. Recuperado de http:// www.ifrs.org/

Kabay, M. E. (2006). The net present value of information security: a paradigm shift for Infosec and E-commerce. Infosec Management Papers, 17, 45- 69.

Lambert, D. M., y Burduroglu, R. (2000). Measuring and selling the value of logistics. The International Journal of Logistic Management, 11. Recuperado de http://ecsocman.edu.ru/images/pubs/2005/12/01/0000242926/article5.pdf

Mascareñas, J. (2000). El Método binomial de valoración de opciones. Universidad Complutense de Madrid. Recuperado de http://www.gacetafinanciera.com/ TEORIARIESGO/VALOREOPCIONES.pdf

Ross, S. A., Westerfield, R. W. y Jaffe, J. F. (2011). Corporate finance (9. ${ }^{a}$ ed.). Estados Unidos: McGraw-Hill.

Smith, A. (1979). Investigación acerca de la naturaleza y causa de la riqueza de las Naciones. México: Fondo de Cultura Económica.

South African Reserve Bank (2012). Prime overdraft rate of banks. Recuperado de http://www.reservebank.co.za/

Tymoigne, E. (2006). Fisher's theory of interest rates and the notion of real: a critique. Working Paper, 483, 106-124. 